\title{
MODELLING OF DATA COMMUNICATION OVER MEDIUM-VOLTAGE POWER DISTRIBUTION LINES
}

The paper is dedicated to different topologies of medium-voltage power distribution lines with respect to their properties influencing the transmission of communication signals. We provide very basic introduction to the problems of signal transmission over these lines, discuss their parameters and examine the typical behaviour and characteristics. We conclude our considerations with some specific recommendations concerning the most advantageous configurations for the purposes of Power Line Communication (PLC). The data transmission capacity is calculated for different topologies and network conditions.

Keywords: PLC; power line communication; Smart Grid; medium-voltage lines.

\section{Introduction}

The concept of Smart Grids in power distribution systems [1] naturally implies the need for transmission of communication signals over medium-voltage lines, and therefore it is necessary to solve some specific problems related to this environment, sometimes substantially different from the issues known in conventional telecommunication lines and cables [2]. In this paper we introduce and analyse some of these problems, with special emphasis on typical topologies and their particular consequences.

\section{State of the Art}

\section{A) Basic model of the line}

Actual resources relevant for the power line network modelling are [3] and [4]. If we want to determine the transmission function of a line composed of several sections with different parameters and containing also taps or other elements, we can express it from the product of matrices describing the individual cascaded sections. We should use the cascade parameters of a two-port network $\mathrm{ABCD}[5]$. The resulting matrix of the cascade will be given by the product of the sectional matrices:

$\mathrm{A}=\mathrm{A}_{1} \cdot \mathrm{A}_{2} \ldots . . \mathrm{A}_{n}=\left[\begin{array}{ll}a(f) & b(f) \\ c(f) & d(f)\end{array}\right]$

where $\mathrm{A}_{1}$ to $\mathrm{A}_{\mathrm{n}}$ are matrices describing the cascaded elements and $a(f)$ to $d(f)$ are the resulting parameters of the entire cascade.
The matrix for section of a homogeneous line with length $l_{i}$, characteristic impedance $Z_{c i}$ and propagation coefficient $\gamma_{i}$ can be calculated using the following formula:

$$
\mathrm{A}_{L i}=\left[\begin{array}{cc}
\left(\cosh \left(\gamma_{i}(f) \cdot l_{i}\right)\right) & \left(Z_{C i}(f) \cdot \sinh \left(\gamma_{i}(f) \cdot l_{i}\right)\right) \\
\left(\frac{\sinh \left(\gamma_{i}(f) \cdot l_{i}\right)}{Z_{C i}(f)}\right) & \left(\cosh \left(\gamma_{i}(f) \cdot l_{i}\right)\right)
\end{array}\right]
$$

\section{B) Modelling of medium-voltage lines}

Let us consider the initial values for modelling of a $22 \mathrm{kV}$ line according to [6], which lists the following primary parameters: $\mathrm{L}=1.9 \cdot 10^{-6} \mathrm{H} / \mathrm{m}, \mathrm{C}=8 \cdot 10^{-12} \mathrm{~F} / \mathrm{m}, \mathrm{R}(200 \mathrm{kHz})=0.03 \Omega / \mathrm{m}$, and $\mathrm{G}(200 \mathrm{kHz})=1.5 \cdot 10^{-6} \mathrm{~S} / \mathrm{m}$. Some other important parameters of British Telecom model (BT9) [7] include: DC resistance $r_{0}=1$; frequency characteristics slope constant $a_{c}=0.0002$; leakage for low frequencies $g_{0}=7.5 \cdot 10^{-10}$; and growth coefficient towards higher frequencies $g_{e}=1$. Then the characteristic impedance $Z_{c}$ can be calculated as:

$Z_{C}=\sqrt{\frac{L}{C}}=\sqrt{\frac{1.9}{8} 10^{6}}=487 \Omega$

and the propagation velocity $v$ as:

$v=\frac{Z_{c}}{L}=256495 \mathrm{~km} / \mathrm{s}$

Local maximums of the attenuation characteristics can be estimated using the following formula:

\footnotetext{
* Jaromir Hrad, Jiri Vodrazka

Department of Telecommunication Engineering, Faculty of Electrical Engineering, Czech Technical University in Prague, Czech Republic E-mail: hrad@fel.cvut.cz
} 


$$
f_{\text {Amax }}=(2 k+1) \cdot \frac{v}{4 l_{b t}}, \text { for } k=0 ; 1 ; 2 ; 3 \ldots
$$

where $l_{b t}$ denotes the length of a bridged tap and $v$ the signal propagation speed on the given line [8] and [9].

\section{Medium-voltage Lines with Branches}

Medium-voltage power distribution network has, in general, a tree topology with many branches (that we refer to as bridged taps, with respect to the common terminology used in the telecommunications area [5]). For the purposes of modelling of the medium-voltage transmission environment for PLC communication we will consider three basic topological configurations:

1. Tree topology,

2. Linear topology with very short taps,

3. Star topology.

These topologies will be compared in a situation with testing length $12.26 \mathrm{~km}$ between the central unit (placed in a HV/MV transformer station) and the most distant modem in a distribution transformer station (DTS). It is assumed that the taps are bridged with a combination of an equivalent impedance of the distribution transformer and a coupling element; so, we can assume that a PLC modem is connected in all DTS nodes.

\section{A) Tree topology}

The first considered topology (Fig. 1) is a tree with taps in nodes No. 2, 4, 6, 8, 9, 12, 14 and 15; their respective lengths are $152 \mathrm{~m}, 2630 \mathrm{~m}, 81 \mathrm{~m}, 1870 \mathrm{~m}, 54 \mathrm{~m}, 289 \mathrm{~m}, 42 \mathrm{~m}$ and $521 \mathrm{~m}$, and the lengths of the individual sections are $385 \mathrm{~m}, 4491 \mathrm{~m}, 27 \mathrm{~m}$, $1751 \mathrm{~m}, 3420 \mathrm{~m}, 1290 \mathrm{~m}$ and $923 \mathrm{~m}$.

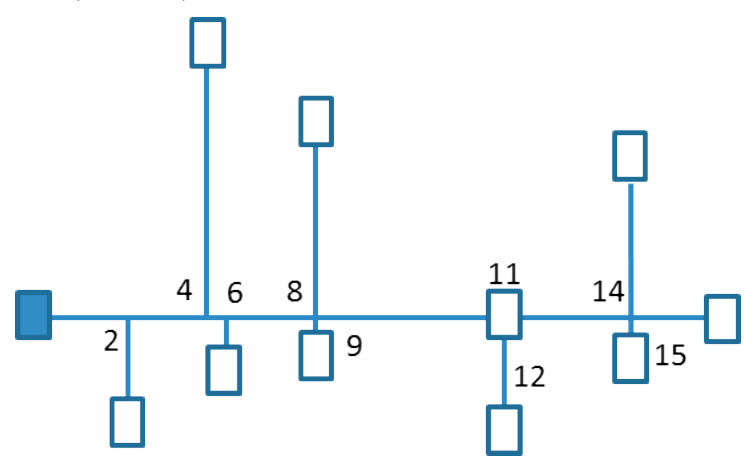

Fig. 1 Configuration \#1

The graph in Fig. 2 shows modelled attenuation for the tree topology (blue curve) compared to a homogeneous line (green curve). In order to compare different situations, let us focus on an identical tree topology, but with extremely increased leakage (10 times higher than in the previous situation), which corresponds to very high values of humidity in rain and fog (see Fig. 3).

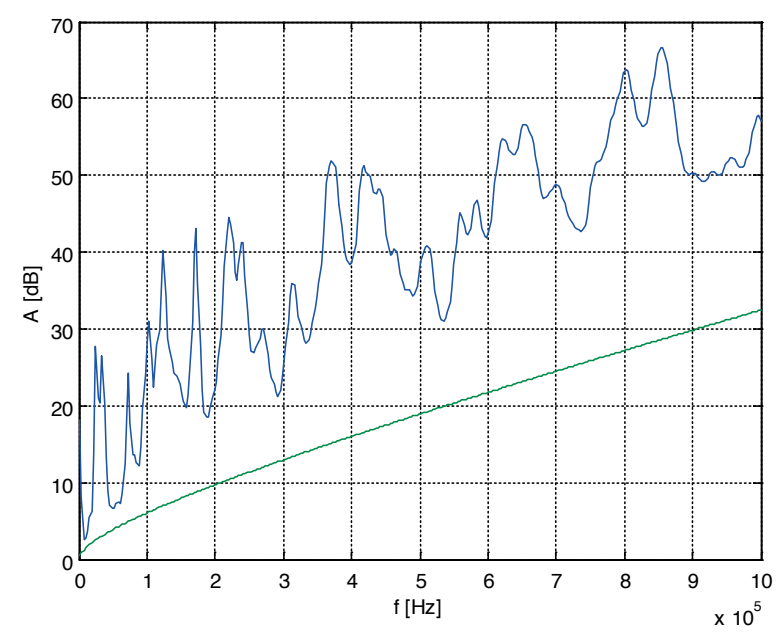

Fig. 2 Frequency dependence of attenuation

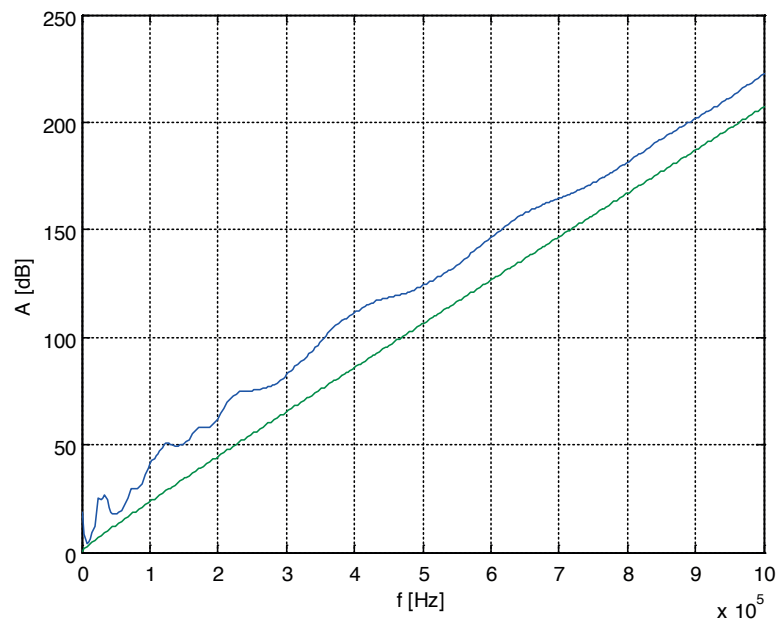

Fig. 3 Frequency dependence of attenuation (for extremely increased leakage)

It can be observed from the frequency dependence of attenuation that thanks to substantial increase of specific attenuation the reflections are attenuated as well (i.e. the maximums are flattened), but - especially in the higher frequency range - the values of attenuation are very high, and therefore the practical usability of such channel for transmission of communication signals is limited to the band up to $100 \mathrm{kHz}$ at most.

C) Linear topology with very short taps

The next considered topology is linear, with short taps leading from the main line to the individual DTS (see Fig. 4). 


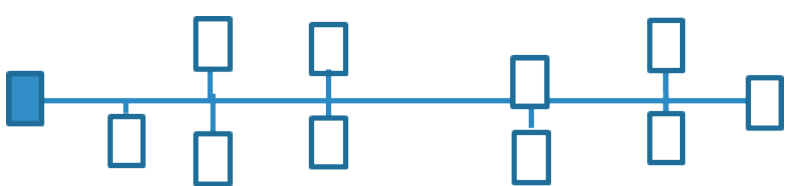

Fig. 4 Configuration \#2 (linear topology with short taps)

The lengths of the respective short taps are $27 \mathrm{~m}, 81 \mathrm{~m}, 54 \mathrm{~m}$, $42 \mathrm{~m}, 16 \mathrm{~m}, 18 \mathrm{~m}, 29 \mathrm{~m}$ and $98 \mathrm{~m}$. The lengths of line sections remain the same as in the case of tree topology. The graph in Fig. 5 shows the modelled attenuation for linear topology with short taps (blue curve) compared to a homogeneous line (green curve).

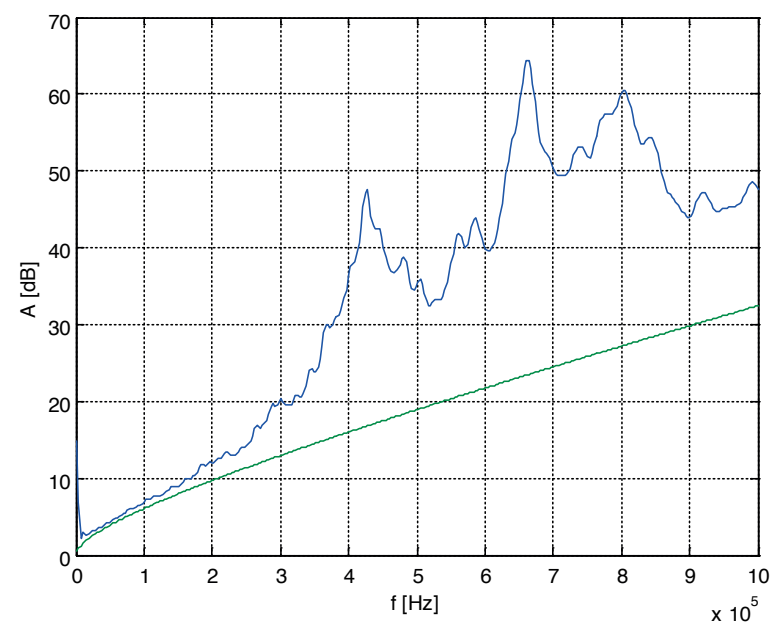

Fig. 5 Dependence of attenuation on frequency (linear topology with short taps)

From the graphs it is clear that up to approximately $300 \mathrm{kHz}$ the taps do not disturb the transmission and the values of attenuation are almost comparable with homogeneous lines. Also the impulse response is much cleaner.

\section{D) Star topology}

The last considered case is a star topology - lines towards the individual DTS begin in the same node. Other transformers may be deployed on these lines, as we can see on the scheme in Fig. 6 .

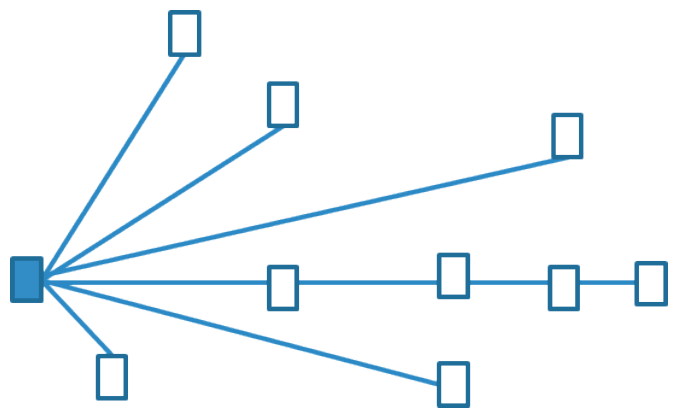

Fig. 6 Configuration \#3 (star topology)
Besides the main examined line $(12.26 \mathrm{~km})$ we consider also five other "beams" of the following lengths: $3853 \mathrm{~m} ; 1527 \mathrm{~m}$; $4491 \mathrm{~m} ; 6762 \mathrm{~m} ; 2630 \mathrm{~m}$. The graph in Fig. 7 shows the modelled attenuation for the star topology (blue curve) compared to a homogeneous line (green curve).

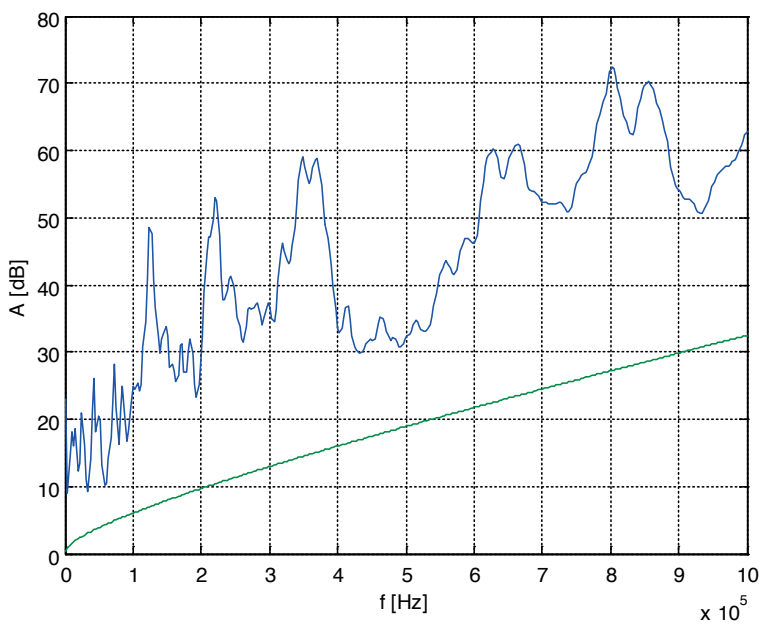

Fig. 7 Frequency dependence of attenuation (star topology)

The modelled dependencies show that their nature is totally different, which is caused by substantial influence of reflections. The most remarkable difference against the previous cases is substantially lower input impedance caused by connecting several lines in parallel. This fact can be used to decrease the internal impedance of modems in the central node of a network with star topology.

\section{Data transmission performance}

We have performed initial modelling, using the tree topology that appears to be the most critical one from the viewpoint of transmission parameters (exhibiting multiple reflections, the most rippled transfer function as well as impulse response). We considered OFDM scheme with $1 \mathrm{kHz}$ subchannel width and QAM modulation, noise margin $\mathrm{NM}=4(6 \mathrm{~dB})$, permissible symbol error rate $10^{-7}$ (Shannon gap 9.5) and signal amplitude $0.1 \mathrm{~V}$ - RMS value per $1 \mathrm{kHz}$ channel (typically corresponding to $10 \mathrm{~V}_{\mathrm{pp}}$ for transmission in the entire considered bandwidth). We did not consider any error correction method. The maximum number of allocated bits is 8 , which corresponds to the maximum number of states for 256-QAM modulation.

Furthermore, we considered background noise typical for industrial environments, taken from low-voltage networks, and interference from LW radio transmitters - DCF77 (time code at $77.5 \mathrm{kHz}$ ) and LW radio broadcast (at $270 \mathrm{kHz}, 153 \mathrm{kHz}$, $183 \mathrm{kHz}, 234 \mathrm{kHz}$ and $225 \mathrm{kHz}$ ). 
Figure 8 shows power spectral density of the considered noise, and Fig. 9 illustrates frequency dependence of attenuation in the channel under optimum transmission conditions for line length (transmitter - receiver) $20 \mathrm{~km}$.

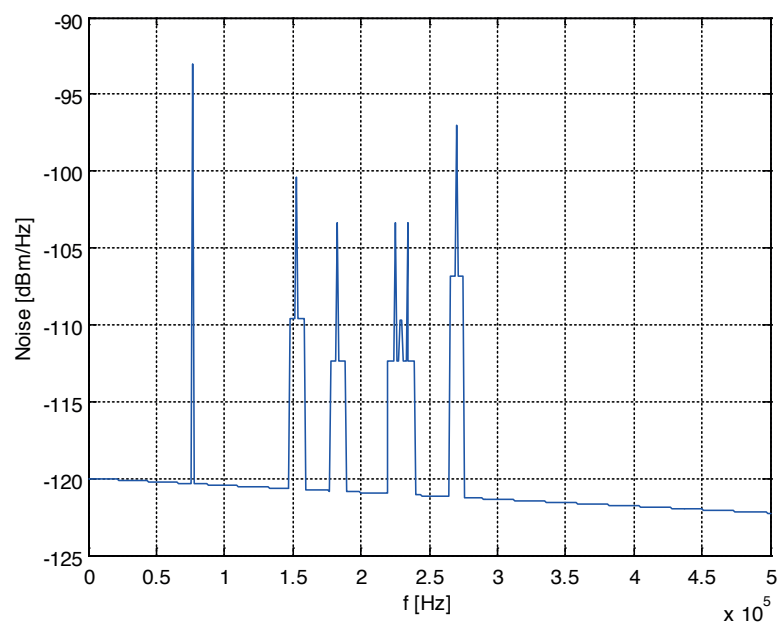

Fig. 8 Power spectral density of noise (star topology)

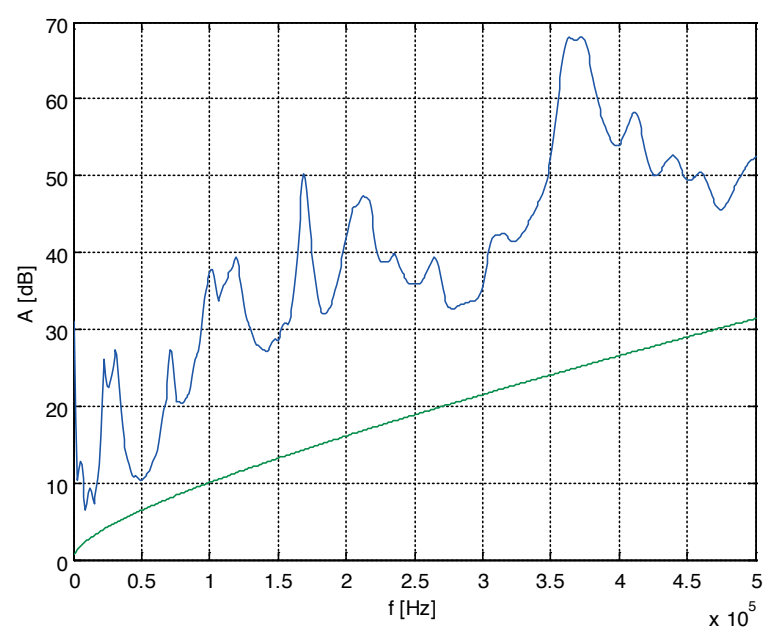

Fig. 9 Frequency dependence of attenuation for $20 \mathrm{~km}$ length (configuration \#1)

The graph in Fig. 10 shows theoretical maximum transmission speed - the blue curve represents line length dependence of the speed for QAM modulation and the green one for OFDM. The graph of bit allocation for OFDM (Fig. 11) shows the usability of different spectral bands (dependence on subchannel sequential number).

If the lower part of the band between 10 and $60 \mathrm{kHz}$ is used for transmission, the resulting data rate is practically independent of the distance. The comparison above does not allow unambiguous decision whether it is more advantageous to use the single-carrier (QAM) or multi-carrier (OFDM) modulation. However, it is positive that acceptable data rates can be achieved even under worsened conditions when the frequencies in the lower part of the band are used.

The current trend in communication systems is the deployment of turbo codes [10] and [11], which leads to lower achievable data rate, but it also rapidly increases system robustness, i.e. noise immunity and acceptable attenuation of the transmission path under extreme conditions.

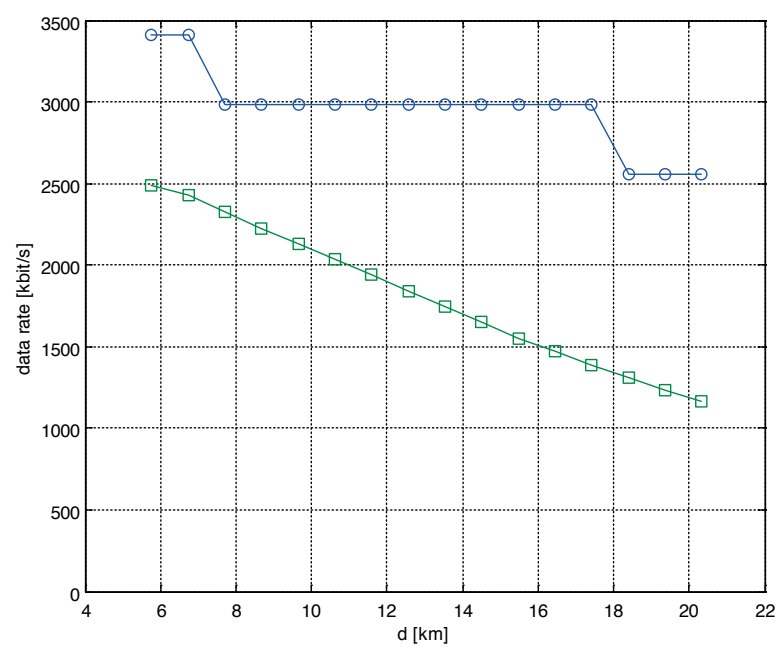

Fig. 10 Dependence of data rate on distance (configuration \#1)

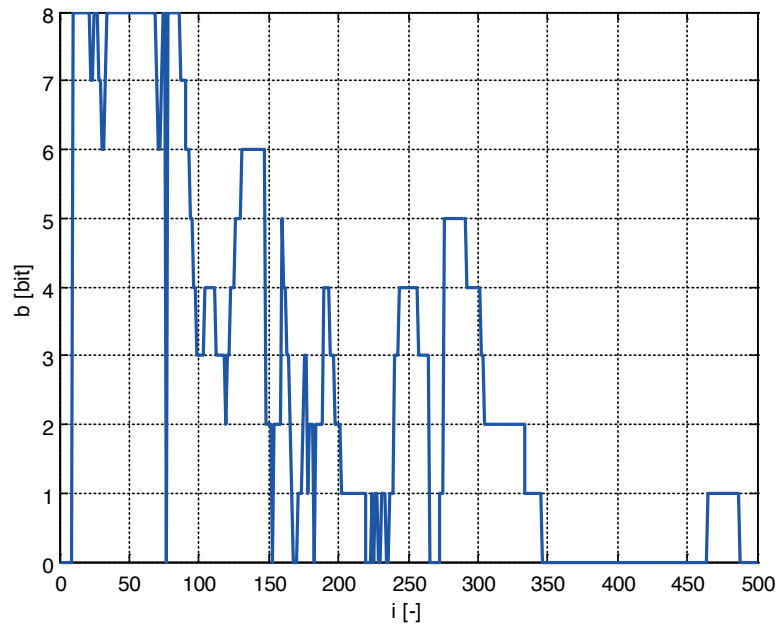

Fig. 11 Example of bit allocation for OFDM transmission format

\section{Conclusion}

The graphs displayed above clearly show that the resulting channel properties for the respective topologies differ substantially, being influenced mainly by reflections. The most remarkable difference of star topology against the other cases consists in 
much lower input impedance, as several lines are connected in parallel; this approach can be used to decrease the internal impedance of a modem in the central node of a star network accordingly.

The most advantageous topology - from the viewpoint of reflections - is the linear one with short taps, where we can identify relatively wide band, suitable for PLC communication, the parameters of which nearly approach those of a homogeneous line.

\section{Acknowledgement}

This paper has originated thanks to grant support obtained from the Technology Agency of the Czech Republic within the project "Research and Development of New-Generation Communication Devices for Transmission Over High-Voltage Power Lines" (TA03011192).

\section{References}

[1] CEPA, L., KOCUR, Z., MULLER, Z.: Migration of the IT Technologies to the Smart Grids. Elektronika ir Elektrotechnika, 2012, vol. 7, No. 123, pp. 123-128. ISSN 1392-1215.

[2] NEVOSAD, M., VODRAZKA, J., LAFATA, P.: Parameters of Twisted Pairs Assessment for Frequency Band up to 1 GHz. Advances in Electrical and Electronic Engineering. 2012, vol. 10, No. 4, pp. 224-227. ISSN 1336-1376.

[3] SANZ, A.: Evolution and Limit of Performance of OFDM-based Narrowband PLC. WSPLC15, $9^{\text {th }}$ Workshop on Power Line Communications, September 2015, Klagenfurt - Austria.

[4] PITTOLO, A., TONELlO, A.: New Results in Channel Modelling. WSPLC15, $9^{\text {th }}$ Workshop on Power Line Communications, September 2015, Klagenfurt - Austria.

[5] VODRAZKA, J., JARES, P., SIMAK, B.: More Accurate Modelling of Digital Subscriber Lines with Inhomogeneity. Communications - Scientific Letters of the University of Zilina. 2013, vol. 15, No. 2A, pp. 129-133. ISSN 1335-4205.

[6] DABAK, A., KIM, I. H., VARADARAJAN, B., PANDE, T.: Channel Modeling for MV/LV AMI Applications in the Frequency Range $<500 \mathrm{kHz}$, Workshop on Power Line Communications WSPLC, 2011.

[7] RAUSCHMAYERM, D. J.: ADSL/VDSL Principles: A Practical and Precise Study of Asymmetric Digital Subscriber Lines and Very High Speed Digital Subscriber Lines. Macmillan Technical Publishing, Indianapolis 1999.

[8] VODRAZKA, J., HRAD, J.: Modeling of a Subscriber Line with Inhomogeneity, 32 ${ }^{\text {nd }}$ Intern. Conference on Telecommunications and Signal Processing (TSP 2009), Dunakiliti, 2009, pp. 79-83, ISBN 978-963-06-7716-5.

[9] HRASNICA, H.: Broadband Powerline Communications Networks. John Wiley \& Sons : Chichester 2004. ISBN 0-470-85741-2

[10] SEDY, J., SILHAVY, P., KRAJSA O., HROUZA, O.: Performance Analysis of Turbo Codes. Communications - Scientific Letters of the University of Zilina, 2013, vol. 15, No. 2A, pp. 167-173. ISSN 1335-4205.

[11] ZAVRTALEK, J., KEKRT, D., HRAD, J.: New Concept of PLC Modems: Multi-carrier System for Frequency Selective Slow-fading Channels Based on Layered SCCC Turbocodes. Radioengineering, 2015, vol. 24, No. 3, pp. 857-872. ISSN 12102512. 